\title{
GLOBAL WEIERSTRASS EQUATIONS OF HYPERELLIPTIC CURVES
}

\author{
QING LIU
}

\begin{abstract}
Given a hyperelliptic curve $C$ of genus $g$ over a number field $K$ and a Weierstrass model $\mathscr{C}$ of $C$ over the ring of integers $\mathcal{O}_{K}$ (i.e. the hyperelliptic involution of $C$ extends to $\mathscr{C}$ and the quotient is a smooth model of $\mathbb{P}_{K}^{1}$ over $\mathcal{O}_{K}$ ), we give necessary and sometimes sufficient conditions for $\mathscr{C}$ to be defined by a global Weierstrass equation. In particular, if $C$ has everywhere good reduction, we prove that it is defined by a global integral Weierstrass equation with invertible discriminant if the class number $h_{K}$ is prime to $2(2 g+1)$, confirming a conjecture of M. Sadek.
\end{abstract}

Let $C$ be a hyperelliptic curve of genus $g \geq 2$ or an elliptic curve over a number field $K$. It is natural to try to represent $C$ with an integral Weierstrass equation (i.e. with coefficients in the ring of integers $\mathcal{O}_{K}$ of $K$ )

$$
y^{2}+Q(x) y=P(x)
$$

in a canonical way. For instance we may require the discriminant of the equation to be minimal in the sense that, at any finite place $v$ of $K$, it divides the discriminant of any Weierstrass equation of $C$ with coefficients in the local ring $\mathcal{O}_{v}$ (Definition 2.4). If $C$ has everywhere good reduction, this means that we are looking for an integral Weierstrass equation with invertible discriminant. It is well known that such a minimal equation exists if $\mathcal{O}_{K}$ is principal, but this is not true in general (see e.g. [11, Corollary 6 or [12, Theorem 1.7, for elliptic curves).

The aim of this work is to study general conditions under which a Weierstrass model, not necessarily minimal, is defined by an integral Weierstrass equation (Definition 2.1). Let us describe briefly the main results. We work more generally over a Dedekind domain $R$ with field of fractions $K$. For a Weierstrass model $\mathscr{C}$ of $C$, the quotient $\mathscr{Z}$ of $\mathscr{C}$ by the hyperelliptic involution is a projective bundle over $S=\operatorname{Spec} R$. For $\mathscr{C}$ to be defined by an integral Weierstrass equation, there are three necessary conditions, see Proposition 2.6.

(a) $\mathscr{Z}$ must be trivial (i.e. $\mathscr{Z} \simeq \mathbb{P}_{S}^{1}$ );

(b) the discriminant ideal $\Delta_{\mathscr{C}} \subseteq R$ must be principal;

(c) if $\omega_{\mathscr{C} / S}$ denotes the dualizing sheaf of $\mathscr{C}$ over $S$, then $\operatorname{det} H^{0}\left(\mathscr{C}, \omega_{\mathscr{C} / S}\right) \simeq R$.

The converse is true when $g$ is not divisible by 4 (see below), but is false in general (Proposition 6.1(d)). These three conditions are independent of each other (see

2020 Mathematics Subject Classification. 11G30, 11G05, 14D10, $14 \mathrm{H} 25$.

Key words and phrases. Hyperelliptic curves, discriminant, Weierstrass equation, good reduction.

I would like to thank heartily Mohammad Sadek for asking the question on the existence of a global minimal equation and for stimulating discussions. I would like to thank Yuri Bilu, Jean-François Jaulent and Jean Gillibert for helpful discussions about Lemma 6.5 I am grateful to one of the anonymous referees for checking thoroughly the first version, finding mistakes and proposing several important improvements, especially in the even genera case. 
$\S$ 6) except when $g=1,2$ or 4 in which case $\Delta_{\mathscr{C}}$ is isomorphic to some power of $\operatorname{det} H^{0}\left(\mathscr{C}, \omega_{\mathscr{C} / S}\right)$ (Corollary 3.10).

Roughly speaking, Condition (a) means there is a rational function $x$ for the integral Weierstrass equation (11) potentially representing $\mathscr{C}$. This condition is automatically satisfied if $\operatorname{Pic}(S)$ is finite of odd order (Proposition 1.4) or if $\mathscr{C}$ is a pointed Weierstrass model (Proposition 4.3) The existence of $y$ for the equation means that the integral closure of $R[x]$ in the function fields $K(C)$ is free over $R[x]$, with a basis $\{1, y\}$. We define the Weierstrass class $[\mathfrak{w}] \in \operatorname{Pic}(S)$ associated to $\mathscr{C}$ (Definition 3.3) and we prove that the existence of an integral Weierstrass equation representing $\mathscr{C}$ is equivalent to the triviality of $[\mathfrak{w}]$ and $\mathscr{Z} \simeq \mathbb{P}_{S}^{1}$ (Proposition 3.4).

The main result of this work is the following theorem. Denote by $f: \mathscr{C} \rightarrow S$ the structure morphism.

Theorem 0.1 (See Theorem 3.11). Let $\mathscr{C}$ be a Weierstrass model of $C$ over $S$. Then $\mathscr{C}$ is defined by an integral Weierstrass equation under any of the following conditions:

(1) $\Delta_{\mathscr{C}}$ is principal, $\operatorname{det} f_{*} \omega_{\mathscr{C} / S}$ is free and either

(a) $g$ is odd, $\mathscr{Z} \simeq \mathbb{P}_{S}^{1}$ or

(b) $g \equiv 2 \bmod 4$;

(2) $\operatorname{Pic}(S)$ is finite of odd order, $\Delta_{\mathscr{C}}$ is principal and $\operatorname{det} f_{*} \omega_{\mathscr{C} / S}$ is free;

(3) $\operatorname{Pic}(S)$ is finite of order prime to $2(2 g+1)$ and $\Delta_{\mathscr{C}}$ is principal;

(4) $\operatorname{Pic}(S)$ is finite of order prime to $2 g$ and $\operatorname{det} f_{*} \omega_{\mathscr{C} / S}$ is free.

In particular, for hyperelliptic curves having everywhere good reduction over a number field, the next corollary answers positively a conjecture of Mohammad Sadek [10. It is well known for elliptic curves.

Corollary 0.2 (See Corollary 3.12). Let $C$ be a hyperelliptic curve of genus $g$ over a number field $K$ having everywhere good reduction. If the class number of $K$ is prime to $2(2 g+1)$, then $C$ is defined by an integral Weierstrass equation with invertible discriminant.

Similar results are given in $\$ 4$ for pointed hyperelliptic curves $(C, P)$ where $P$ is a rational Weierstrass point of $C$. Finally in 6 , we construct examples of $\mathscr{C}$ satisfying various nice properties but not defined by integral (or pointed integral) Weierstrass equations.

Settings In all this paper, $R$ is a Dedekind domain, $K=\operatorname{Frac}(R), S=\operatorname{Spec} R$ and $C$ is a hyperelliptic curve over $K$ (that is, $C$ is smooth projective geometrically connected over $K$ and admits a finite separable morphism $C \rightarrow \mathbb{P}_{K}^{1}$ of degree 2) of genus $g \geq 2$ or an elliptic curve over $K$.

\section{Twisted PROJECTIVE LINES}

Let $\phi: \mathscr{Z} \rightarrow S$ be a smooth projective scheme with generic fiber isomorphic to $\mathbb{P}_{K}^{1}$. This is a twisted $\mathbb{P}_{S}^{1}$ (see [9], 3.2). It is the projective bundle over $S$ defined by a locally free rank 2 vector bundle on $S$.

In this section we study the sections of $\mathscr{Z}$ and also give a sufficient condition for $\mathscr{Z}$ to be isomorphic to $\mathbb{P}_{S}^{1}$. We often consider sections of $\mathscr{Z}$ as effective Cartier divisors on $\mathscr{Z}$. Then $\mathcal{O}_{\mathscr{Z}}(D)$ is a subsheaf of the function field $K(\mathscr{Z})$. 
Definition 1.1 Let $D \in \mathscr{Z}(S)$ be a section. We will say that $D$ is elementary if $\phi_{*} \mathcal{O}_{\mathscr{Z}}(D)$ is free over $\mathcal{O}_{S}$.

Lemma 1.2. Let $D \in \mathscr{Z}(S)$. Let $F=\phi_{*} \mathcal{O}_{\mathscr{Z}}(D)$.

(1) The invertible sheaf $\mathcal{O}_{\mathscr{Z}}(D)$ is very ample, $F$ is locally free of rank 2 over $\mathcal{O}_{S}$, the morphism $i: \mathscr{Z} \rightarrow \mathbb{P}(F)$ associated to $\mathcal{O}_{\mathscr{Z}}(D)$ is an isomorphism and we have

$$
i^{*} \mathcal{O}_{\mathbb{P}(F)}(1) \simeq \mathcal{O}_{\mathscr{Z}}(D)
$$

(2) The following properties are equivalent:

(a) $D$ is elementary;

(b) $\operatorname{det} F \simeq \mathcal{O}_{S}$;

(c) There exists an isomorphism $\theta: \mathscr{Z} \rightarrow \mathbb{P}_{S}^{1}$ such that $\mathcal{O}_{\mathscr{Z}}(D) \simeq \theta^{*} \mathcal{O}_{\mathbb{P}_{S}^{1}}(1)$.

(3) Let $D^{\prime} \in \mathscr{Z}(S)$. Then there exists a unique $M \in \operatorname{Pic}(S)$ such that

$$
\mathcal{O}_{\mathscr{Z}}\left(D^{\prime}\right) \simeq \mathcal{O}_{\mathscr{Z}}(D) \otimes \phi^{*} M
$$

We then have

$$
\phi_{*} \mathcal{O}_{\mathscr{Z}}\left(D^{\prime}\right) \simeq F \otimes M, \quad \operatorname{det} \phi_{*} \mathcal{O}_{\mathscr{Z}}\left(D^{\prime}\right) \simeq(\operatorname{det} F) \otimes M^{\otimes 2} .
$$

(4) For any $M \in \operatorname{Pic}(S)$, there is a section $D^{\prime} \in \mathscr{Z}(S)$ such that

$$
\mathcal{O}_{\mathscr{Z}}\left(D^{\prime}\right) \simeq \mathcal{O}_{\mathscr{Z}}(D) \otimes \phi^{*} M
$$

Proof. (1) This is true because it is true locally on $S$.

(2) Obviously (c) $\Rightarrow$ (a) $\Rightarrow$ (b). We have (b) $\Rightarrow$ (a) because $F \simeq \mathcal{O}_{S} \oplus \operatorname{det} F, R$ being a Dedekind domain.

(a) $\Rightarrow$ (c). Compose the isomorphism $i: \mathscr{Z} \simeq \mathbb{P}(F)$ with an isomorphism $\mathbb{P}(F) \simeq \mathbb{P}_{S}^{1}$ to get the desired isomorphism $\theta$. Then $\theta^{*} \mathcal{O}_{\mathbb{P}_{S}^{1}}(1) \simeq \mathcal{O}_{\mathscr{Z}}(D)$ by the isomorphism of Part (1).

(3) For any noetherian connected scheme $T$ and any locally free $\mathcal{O}_{T}$-module $G$ of finite rank, it is known that there is a canonical isomorphism

$$
\operatorname{Pic}(\mathbb{P}(G)) \simeq\left\langle\mathcal{O}_{\mathbb{P}(G)}(1)\right\rangle \times \operatorname{Pic}(T)
$$

(see 5], II, Remarque 4.2.7, or 4, Proposition 8.4(a)). This implies the existence and uniqueness of $M$. The second part comes from the projection formula (7], Proposition 5.2.32) and the fact that $F$ is locally free of rank 2.

(4) We work over $\mathbb{P}(F)$ with $\mathcal{O}_{\mathbb{P}(F)}(1) \simeq \mathcal{O}_{\mathbb{P}(F)}(D)$. Let $H=M^{\vee}$ be the dual of $M$. Using the structure of the projective modules over Dedekind domains, $H$ can be injected into $F$ with locally free quotient of rank 1 . Then $H$ induces a section $D^{\prime}$ of $\mathbb{P}(F)$ ([5], II.4.2.3). As an effective Cartier divisor on $\mathbb{P}(F), D^{\prime}$ is defined by the sheaf of homogeneous ideals $H \mathcal{O}_{\mathbb{P}(F)}$. One can check that $\mathcal{O}_{\mathbb{P}(F)}\left(-D^{\prime}\right) \otimes \mathcal{O}_{\mathbb{P}(F)}(1) \simeq$ $\phi^{*} H$, thus the desired isomorphism.

Example 1.3 (1) If $R$ is a PID, then all sections of $\mathscr{Z}$ are elementary.

(2) On $\mathbb{P}_{S}^{1}$ with a parameter $x$, a typical elementary section is the pole divisor $\operatorname{div}_{\infty}(x)$ of $x$ with $\mathcal{O}_{\mathbb{P}_{S}^{1}}\left(\operatorname{div}_{\infty}(x)\right) \simeq \mathcal{O}_{\mathbb{P}_{S}^{1}}(1)$.

In view of Lemma $1.2(2)$, when $\mathscr{Z}$ has an elementary section $D$, we will often think about the pair $(\mathscr{Z}, D)$ as $\left(\mathbb{P}_{S}^{1}, \operatorname{div}_{\infty}(x)\right)$ where $x$ is some parameter of $\mathbb{P}_{S}^{1}$. 
Proposition 1.4. We have $\mathscr{Z} \simeq \mathbb{P}_{S}^{1}$ if and only if for some $D \in \mathscr{Z}(S)$, $\operatorname{det} \phi_{*} \mathcal{O}_{\mathscr{Z}}(D)$ is a square in $\operatorname{Pic}(S)$. In particular, if the map

$$
\operatorname{Pic}(S) \rightarrow \operatorname{Pic}(S), \quad x \mapsto x^{2}
$$

is surjective (e.g. if $\operatorname{Pic}(S)$ has finite and odd order), then $\mathscr{Z} \simeq \mathbb{P}_{S}^{1}$.

Proof. Let $D \in \mathscr{Z}(S)$ and $F=\phi_{*} \mathcal{O}_{\mathscr{Z}}(D)$. We have $\mathscr{Z} \simeq \mathbb{P}(F)$ by Lemma 1.2(1). By Lemma 1.5 below, $\mathscr{Z} \simeq \mathbb{P}_{S}^{1}$ if and only if $F \simeq M \oplus M$ for some $M \in \operatorname{Pic}(S)$. The existence of such an isomorphism implies that $\operatorname{det} F \simeq M^{\otimes 2}$ is a square in $\operatorname{Pic}(S)$. Conversely, if $\operatorname{det} F \simeq M^{\otimes 2}$ for some $M \in \operatorname{Pic}(S)$, replacing $F$ by $F \otimes M^{\vee}$ (which does not change $\mathbb{P}(F)$ ), we can suppose that $\operatorname{det} F \simeq \mathcal{O}_{S}$. Therefore $F$ is free (Lemma 1.2(2)) and $\mathscr{Z} \simeq \mathbb{P}_{S}^{1}$.

Lemma 1.5. Let $F, G$ be two locally free finite rank sheaves on a noetherian scheme $T$. Then the projective bundles $\mathbb{P}(F)$ and $\mathbb{P}(G)$ are $T$-isomorphic if and only if $F \simeq G \otimes_{\mathcal{O}_{T}} M$ for some $M \in \operatorname{Pic}(T)$.

Proof. The if part is a general fact ([5], II.4.1.4). To prove the converse, we can suppose that $T$ is connected. We have a commutative diagram

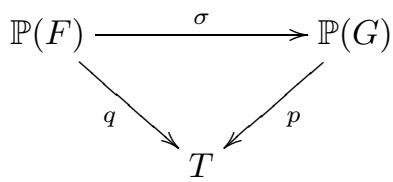

with an isomorphism $\sigma$. Using the structure of $\operatorname{Pic}(\mathbb{P}(G)$ ) (see Isomorphism (2) in the proof of Lemma $1.2(3))$, there exists a $M \in \operatorname{Pic}(T)$ such that $\sigma_{*} \mathcal{O}_{\mathbb{P}(F)}(1)=$ $\mathcal{O}_{\mathbb{P}(G)}(1) \otimes p^{*} M$. Taking $p_{*}$ and using the projection formula ([7], Proposition 5.2 .32 ), we find

$$
q_{*} \mathcal{O}_{\mathbb{P}(F)}(1)=p_{*} \mathcal{O}_{\mathbb{P}(G)}(1) \otimes M .
$$

But $q_{*} \mathcal{O}_{\mathbb{P}(F)}(1) \simeq F\left(\underline{13}\right.$, Example 27.21.3) and $p_{*} \mathcal{O}_{\mathbb{P}(G)}(1) \simeq G$, the only if part is proved.

\section{WEIERSTRASS MODELS AND DISCRIMINANT IDEALS}

In this section we gather some basic definitions and facts on Weierstrass equations and Weierstrass models.

A model of $C$ over $S$ is a normal projective scheme $f: \mathscr{C} \rightarrow S$ whose generic fiber $\mathscr{C}_{K}$ is isomorphic to $C$. A model $\mathscr{C}$ is a Weierstrass model if the hyperelliptic involution on $C$ extends to an $S$-involution $\sigma$ on $\mathscr{C}$ and if the quotient

$$
\mathscr{Z}:=\mathscr{C} /\langle\sigma\rangle
$$

is smooth over $S$. The latter is then a smooth model of $\mathbb{P}_{K}^{1}$ over $S$. Equivalently, a Weierstrass model is the normalization of a smooth model $\mathscr{Z}$ of $\mathbb{P}_{K}^{1}$ in the canonical degree 2 morphism $C \rightarrow \mathbb{P}_{K}^{1}$.

Definition 2.1 A Weierstrass equation of $C$ is an equation of the form

$$
y^{2}+Q(x) y=P(x), \quad P(x), Q(x) \in K[x]
$$

with $\operatorname{deg} Q(x) \leq g+1, \operatorname{deg} P(x) \leq 2 g+2$ such that the corresponding affine curve is isomorphic to an open subscheme of $C$. Such an equation is said to be integral 
over $R$ if moreover $P(x), Q(x) \in R[x]$. If an integral Weierstrass equation (3) of $C$ defines a normal affine scheme

$$
\operatorname{Spec} R[x, y] /\left(y^{2}+Q(x) y-P(x)\right)
$$

(see [6], $\S 3$, Lemme 2 for a criterion of normality), then embedding Spec $R[x]$ in $\mathbb{P}_{S}^{1}$ and taking the normalization of $\mathbb{P}_{S}^{1}$ in the degree 2 morphism $C \rightarrow \mathbb{P}_{K}^{1}$ will give a Weierstrass model $\mathscr{C}$. Such a Weierstrass model is said to be defined by the integral Weierstrass equation (3). The scheme $\mathscr{C}$ is then obtained by glueing the two affine schemes:

$$
\text { Spec } R[x, y] /\left(y^{2}+Q(x) y-P(x)\right), \operatorname{Spec} R[t, z] /\left(z^{2}+t^{g+1} Q(1 / t) z-t^{2 g+2} P(1 / t)\right)
$$

via the identifications $t=1 / x, z=y / x^{g+1}$.

Remark 2.2 Roughly speaking, for $\mathscr{C}$ to be defined by an integral Weierstrass equation, there are two conditions: $\mathscr{Z} \simeq \mathbb{P}_{S}^{1}$ (there exists an $x$ ) and the quotient by $R[x]$ of the integral closure of $R[x]$ in $K(C)$ is free (there exists an $y$ ).

Lemma 2.3. Suppose that $R$ is a PID. Then all Weierstrass models are defined by integral Weierstrass equations.

Proof. This is well known. One can for instance argue as follows: by Proposition 1.4, $\mathscr{Z} \simeq \mathbb{P}_{S}^{1}$. Choose any (automatically elementary) section $D \in \mathscr{Z}(S)$ and argue as in the proof of Proposition 3.4

Definition 2.4 Now we define the discriminant ideal and minimal Weierstrass models. Let $\mathscr{C}$ be a Weierstrass model over $S$. For any closed point $s \in S$, $\mathscr{C} \times{ }_{S}$ Spec $\mathcal{O}_{S, s}$ is then defined by a Weierstrass equation over the PID $\mathcal{O}_{S, s}$. The discriminant of the equation $([6], \S 2)$ generates an ideal $\Delta_{s}$ of $\mathcal{O}_{S, s}$ independent on the choice of the equation. We have $\Delta_{s}=\mathcal{O}_{S, s}$ if and only if $\mathscr{C}$ is smooth at $s$. The discriminant ideal $\Delta_{\mathscr{C}}$ of $\mathscr{C}$ is the ideal $\prod_{s} \Delta_{s}$. We have $\Delta_{\mathscr{C}}=\mathcal{O}_{S}$ if and only if $\mathscr{C}$ is smooth over $S$ (then $C$ has everywhere good reduction). We say that $\mathscr{C}$ is a minimal Weierstrass model if for all $s, \Delta_{s}$ divides the discriminant of any integral Weierstrass equation of $C$ over $\mathcal{O}_{S, s}$.

Remark 2.5 The curve $C$ always has minimal Weierstrass models. Indeed, $C$ has a smooth projective model over a dense open subset $U$ of $S$. Then one can glue this model with minimal Weierstrass models at the points of $S \backslash U$. Note that in general the minimal Weierstrass models are not unique (unless $C$ has good reduction everywhere). However, up to $S$-isomorphisms, there are only finitely many such models for a given $C$ (use [6], $\S 8$, Corollary 4).

Given a Weierstrass model $\mathscr{C}$, there are some natural conditions for $\mathscr{C}$ to be defined by an integral Weierstrass equation. As $f: \mathscr{C} \rightarrow S$ is locally a complete intersection, its dualizing sheaf $\omega_{\mathscr{C} / S}$ is invertible and $f_{*} \omega_{\mathscr{C} / S}$ is locally free of rank $g$ over $S$.

Proposition 2.6. If a Weierstrass model $\mathscr{C}$ is defined by an integral Weierstrass equation, then

(a) $\mathscr{Z} \simeq \mathbb{P}_{S}^{1}$;

(b) $\Delta_{\mathscr{C}}$ is a principal ideal of $R$;

(c) $\operatorname{det} f_{*} \omega_{\mathscr{C} / S}$ is free. 
Proof. The first two conditions are clear. If $\mathscr{C}$ is defined by an integral Weierstrass equation (3), then one can check exactly as over a ground field (see e.g. 7], Proposition 7.4.26) that $f_{*} \omega_{\mathscr{C} / S}$ is free over $R$ with

$$
\frac{x^{i} d x}{2 y+Q(x)}, \quad 0 \leq i \leq g-1
$$

as a basis. So $\operatorname{det} f_{*} \omega_{\mathscr{C} / S}$ is free.

\section{Weierstrass Class of a Weierstrass MOdel}

We fix a Weierstrass model $\mathscr{C}$ and we look for conditions ensuring it is defined by an integral Weierstrass equation. Recall that $f: \mathscr{C} \rightarrow S$ is the structure morphism. Denote by $\pi: \mathscr{C} \rightarrow \mathscr{Z}$ the quotient morphism by the hyperelliptic involution and by $\phi: \mathscr{Z} \rightarrow S$ the structure morphism:

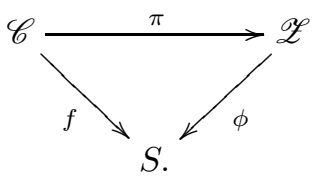

The morphism $\pi$ is finite, locally free of degree 2 , and we have an exact sequence

$$
0 \rightarrow \mathcal{O}_{\mathscr{Z}} \rightarrow \pi_{*} \mathcal{O}_{\mathscr{C}} \rightarrow \mathcal{L}:=\pi_{*} \mathcal{O}_{\mathscr{C}} / \mathcal{O}_{\mathscr{Z}} \rightarrow 0 \text {. }
$$

Lemma 3.1. Let $D \in \mathscr{Z}(S)$ be a section of $\mathscr{Z}$ over $S$. Let $\mathcal{L}$ be as above. Then there exists a unique $N \in \operatorname{Pic}(S)$, depending on $D$, such that

$$
\mathcal{L} \otimes \mathcal{O}_{\mathscr{Z}}((g+1) D) \simeq \phi^{*} N .
$$

Proof. For any $s \in S$, we let $U \ni s$ be an affine open neighborhood such that $\mathscr{C}_{U}$ is defined by an integral Weierstrass equation (Lemma 2.3)

$$
y_{U}^{2}+Q_{U}\left(x_{U}\right) y_{U}=P_{U}\left(x_{U}\right)
$$

(thus $\mathscr{Z}_{U} \simeq \mathbb{P}_{U}^{1}$ with parameter $x_{U}$ ) and that $\left.D\right|_{\mathscr{Z}_{U}}$ is the pole divisor of $x_{U}$. Consider the covering of $\mathscr{Z}_{U}$ by

$$
W:=\operatorname{Spec} \mathcal{O}_{S}(U)\left[x_{U}\right], \quad V:=\operatorname{Spec} \mathcal{O}_{S}(U)\left[1 / x_{U}\right] .
$$

Then

$$
\left(\pi_{*} \mathcal{O}_{\mathscr{C}}\right)(W)=\mathcal{O}_{\mathscr{Z}}(W) \oplus \mathcal{O}_{\mathscr{Z}}(W) y_{U}=\mathcal{O}_{\mathscr{Z}}(W) \oplus \mathcal{O}_{\mathscr{Z}}(-(g+1) D)(W) y_{U}
$$

and

$$
\left(\pi_{*} \mathcal{O}_{\mathscr{C}}\right)(V)=\mathcal{O}_{\mathscr{Z}}(V) \oplus \mathcal{O}_{\mathscr{Z}}(V)\left(y_{U} / x_{U}^{g+1}\right)=\mathcal{O}_{\mathscr{Z}}(V) \oplus \mathcal{O}_{\mathscr{Z}}(-(g+1) D)(V) y_{U} .
$$

Therefore

$$
\left.\pi_{*} \mathcal{O}_{\mathscr{C}}\right|_{\mathscr{Z}_{U}}=\mathcal{O}_{\mathscr{Z}_{U}} \oplus \mathcal{O}_{\mathscr{Z}_{U}}(-(g+1) D) y_{U}
$$

and

$$
\left.\left.\mathcal{L}\right|_{\mathscr{L}_{U}} \simeq \mathcal{O}_{\mathscr{Z}}(-(g+1) D)\right|_{\mathscr{L}_{U}} .
$$

This proves the existence of $N$. The uniqueness of $N$ up to isomorphism comes from the fact that $N \simeq \phi_{*} \phi^{*} N$. 
Remark 3.2 (Dependence on $D$ ) Denote by $F=\phi_{*} \mathcal{O}_{\mathscr{Z}}(D)$. Let $D^{\prime} \in \mathscr{Z}(S)$ be another section with $F^{\prime}=\phi_{*} \mathcal{O}_{\mathscr{Z}}\left(D^{\prime}\right)$ and corresponding $N^{\prime} \in \operatorname{Pic}(S)$ as in Lemma 3.1. Then by Lemma 1.2(3), $\mathcal{O}_{\mathscr{Z}}\left(D^{\prime}\right) \simeq \mathcal{O}_{\mathscr{Z}}(D) \otimes \phi^{*} M$ for a unique $M \in \operatorname{Pic}(S)$ and we have

$$
N^{\prime} \simeq N \otimes M^{\otimes(g+1)}, \quad \operatorname{det} F^{\prime} \simeq(\operatorname{det} F) \otimes M^{\otimes 2} .
$$

In particular,

$$
G_{\mathscr{C}, 2}:=N^{\otimes 2} \otimes\left(\operatorname{det} F^{\vee}\right)^{\otimes(g+1)} \in \operatorname{Pic}(S)
$$

is independent of the choice of $D$. If $g$ is odd, then $G_{\mathscr{C}, 2}$ is the square of

$$
G_{\mathscr{C}, 1}:=N \otimes\left(\operatorname{det} F^{\vee}\right)^{\otimes(g+1) / 2} \in \operatorname{Pic}(S)
$$

and the latter is also independent on the choice of $D$.

Definition 3.3 Let $\mathscr{C}$ be a Weierstrass model. We define the Weierstrass class $[\mathfrak{w}] \in \operatorname{Pic}(S)$ of $\mathscr{C}$ as the class of $G_{\mathscr{C}, 1}$ (resp. $G_{\mathscr{C}, 2}$ ) when $g$ is odd (resp. even).

Proposition 3.4. Let $f: \mathscr{C} \rightarrow S$ be a Weierstrass model of $C$. Then $\mathscr{C}$ can be defined by an integral Weierstrass equation if and only if $\mathscr{Z} \simeq \mathbb{P}_{S}^{1}$ and $[\mathfrak{w}]$ is trivial.

Proof. Step (1) Suppose there exists an elementary section $D \in \mathscr{Z}(S)$ such that the corresponding $N$ is trivial (Lemma 3.1). Let us show the existence of an integral Weierstrass equation defining $\mathscr{C}$.

We identify $(\mathscr{Z}, D)$ with $\left(\mathbb{P}_{S}^{1}, \operatorname{div}_{\infty}(x)\right)$ for some parameter $x$ of $\mathbb{P}_{S}^{1}$. Let $y \in$ $K(C)$ be a basis of $\mathcal{L} \otimes \mathcal{O}_{\mathscr{Z}}((g+1) D)$. Over $W:=\operatorname{Spec} R[x]$, we have $\mathcal{L} \otimes \mathcal{O}_{\mathscr{Z}}((g+$ 1) $D)(W)=R[x] y$. This means that

$$
\mathcal{O}_{\mathscr{C}}\left(\pi^{-1}(W)\right)=R[x] \oplus R[x] y .
$$

So there exist $Q(x), P(x) \in R[x]$ such that $y^{2}+Q(x) y=P(x)$. It remains to bound the degrees of $Q(x)$ and $P(x)$. Over $V:=\operatorname{Spec} R[1 / x]$, we have

$$
\mathcal{L}(V)=\mathcal{O}_{\mathscr{Z}}(-(g+1) D)(V) y=R[1 / x]\left(y / x^{g+1}\right),
$$

which implies that $y / x^{g+1} \in \mathcal{O}_{\mathscr{C}}\left(\pi^{-1}(V)\right)$, hence integral over $R[1 / x]$. Therefore $Q(x) / x^{g+1}, P(x) / x^{2 g+2} \in R[1 / x]$ and $\operatorname{deg} Q(x) \leq g+1, \operatorname{deg} P(x) \leq 2 g+2$. The pair $x, y$ gives an integral Weierstrass equation for $\mathscr{C}$.

Step (2), "only if" part. Suppose that $\mathscr{C}$ is defined by an integral Weierstrass equation as Equation (3). We have $\mathscr{Z} \simeq \mathbb{P}_{S}^{1}$ with parameter $x$. The computations in the proof of Lemma 3.1 show that for $D=\operatorname{div}_{\infty}(x)$, the corresponding $N$ is free. As $D$ is elementary, [ $\mathfrak{w}]$ is trivial.

Step (3), "if" part. Suppose that $\mathscr{Z} \simeq \mathbb{P}_{S}^{1}$ and [w] is trivial. Let $x$ be a parameter of $\mathbb{P}_{S}^{1}$ and let $D=\operatorname{div}_{\infty}(x)$. If $g$ is odd, then $N \simeq[\mathfrak{w}]$ is trivial. It follows from Step (1) that $\mathscr{C}$ is defined by an integral Weierstrass equation.

Suppose now that $g$ is even. Then $N^{\otimes 2} \simeq[\mathfrak{w}]$ is trivial. By Lemma 1.2(4), there exists a section $D^{\prime} \in \mathscr{Z}(S)$ such that

$$
\mathcal{O}_{\mathscr{Z}}\left(D^{\prime}\right) \simeq \mathcal{O}_{\mathscr{Z}}(D) \otimes \phi^{*} N \text {. }
$$

This implies that $\operatorname{det} \phi_{*} \mathcal{O}_{\mathscr{Z}}\left(D^{\prime}\right) \simeq N^{\otimes 2}$ and $N^{\prime} \simeq N \otimes N^{\otimes(g+1)}$ are free, therefore $\mathscr{C}$ is defined by an integral Weierstrass equation, again by applying Step (1) to $D^{\prime}$.

Remark 3.5 In view of this proposition, our Remarque 6 in [6], page 4584, is very likely to be wrong. 
Remark 3.6 (Equations defining the same Weierstrass model) Suppose that $\mathscr{C}$ is defined by an integral Weierstrass equation $y^{2}+Q(x) y=P(x)$.

(a) If $g$ is even or if $\operatorname{Pic}(S)[2]=\{1\}$, then any other integral Weierstrass equation $z^{2}+G(u) z=F(u)$ of $\mathscr{C}$ is related to the previous one by

$$
u=\frac{a x+b}{c x+d}, \quad \text { with }\left(\begin{array}{ll}
a & b \\
c & d
\end{array}\right) \in \mathrm{Gl}_{2}(R)
$$

and

$$
z=\frac{e y+H(x)}{(c x+d)^{g+1}}, \quad e \in R^{*}, H(x) \in R[x], \operatorname{deg} H(x) \leq g+1 .
$$

Indeed, let $D^{\prime}=\operatorname{div}_{\infty}(u)$ with corresponding $F^{\prime}=\phi_{*} \mathcal{O}_{\mathscr{Z}}\left(D^{\prime}\right) \simeq \mathcal{O}_{S}^{2}$ and $N^{\prime}$ as in Lemma 3.1. As seen in Step (2) of the proof of Proposition 3.4. we have $N^{\prime} \simeq \mathcal{O}_{S}$. Let $M$ be as in Remark [3.2, then $M^{\otimes 2} \simeq \mathcal{O}_{S}$ and $M^{\otimes(g+1)} \simeq \mathcal{O}_{S}$. So $M \simeq \mathcal{O}_{S}$, and this implies the relation between $x$ and $u$. The condition on $z$ comes from the fact that $(c x+d)^{g+1} z \in K(C)$ is integral over $R[x]$ and that both equations give the same discriminant ideal $\Delta_{\mathscr{C}}$.

(b) On the opposite, suppose that $g$ is odd and that there exists $M \in \operatorname{Pic}(S)$ of order exactly 2. We take a section $D^{\prime} \in \mathscr{Z}(S)$ as in Lemma 1.2(4). Then $D^{\prime}$ is elementary as $M^{\otimes 2} \simeq \mathcal{O}_{S}$. Moreover the corresponding $N^{\prime} \simeq N \otimes M^{\otimes g+1} \simeq$ $\mathcal{O}_{S}$. By Step (1) in the proof of Proposition 3.4 if $D^{\prime}=\operatorname{div}_{\infty}(u)$, then $\mathscr{C}$ is defined by an integral Weierstrass equation $z^{2}+G(u) z=F(u)$. But $u, x \in$ $K(\mathscr{Z})$ are not related by the action of $\mathrm{Gl}_{2}(R)$.

Note that this phenomena does not happen for pointed Weierstrass models (Definition 4.11) as we then have $\operatorname{div}_{\infty}(u)=\operatorname{div}_{\infty}(x)$, so $u=a x+b$ for some $a \in R^{*}$ and $b \in R$.

Now we relate the Weierstrass class to some other classes attached to $\mathscr{C}$.

Lemma 3.7 (Approximation of local equations). Let $\mathscr{C}$ be a Weierstrass model of $C$ over $S$. Let $D \in \mathscr{Z}(S)$. Let us fix

(a) a Weierstrass equation of $C$ over $K$ :

$$
y^{2}+Q(x) y=P(x)
$$

with $x$ having its pole at $D_{K}$ and

(b) a non-empty open subset $U \subset S$.

Then there exist $r \in \mathcal{O}_{S}(U), h(x) \in \mathcal{O}_{S}(U)[x]$ of degree $\leq g+1$ and, for all $s \in S \backslash U$, an integral Weierstrass equation

$$
y_{s}^{2}+Q_{s}\left(x_{s}\right) y_{s}=P_{s}\left(x_{s}\right)
$$

for $\mathscr{C} \times{ }_{S} \operatorname{Spec} \mathcal{O}_{S, s}$ with

$$
x-r=a_{s} x_{s}, \quad y-h(x)=b_{s} y_{s}, \quad a_{s}, b_{s} \in K^{*} .
$$

Proof. For each $s \in S \backslash U$, there exists an integral Weierstrass equation

$$
y_{s}^{2}+Q_{s}\left(x_{s}\right) y_{s}=P_{s}\left(x_{s}\right)
$$

for $\mathscr{C} \times{ }_{S} \operatorname{Spec} \mathcal{O}_{S, s}$ with $x_{s}$ having its pole at $D_{K}$. So there exist $a_{s}, b_{s} \in K^{*}$, $r_{s} \in K, h_{s}(x) \in K[x]$ of degree $\leq g+1$ such that

$$
x=a_{s} x_{s}+r_{s}, \quad y=b_{s} y_{s}+h_{s}(x) .
$$


By the approximation property in Dedekind domains, there exist $r \in \mathcal{O}_{S}(U), h(x) \in$ $\mathcal{O}_{S}(U)[x]$ of degree $\leq g+1$ such that

$$
r-r_{s} \in a_{s} \mathcal{O}_{S, s}, \quad h(x)-h_{s}(x) \in b_{s} \mathcal{O}_{S, s}[x]
$$

for all $s \in S \backslash U$. We obtain the desired result after replacing $x_{s}$ with $x_{s}-\left(r-r_{s}\right) / a_{s}$ and $y_{s}$ with $y_{s}-\left(h(x)-h_{s}(x)\right) / b_{s}$.

Definition 3.8 Keep the settings of Lemma 3.7. Choose an open subset $U$ such that the equation (8) has good reduction over $U$ and defines $\mathscr{C}_{U}$ (so $\left.D\right|_{\mathscr{Z}_{U}}$ is the pole divisor of $\left.x\right|_{\mathscr{Z}_{U}}$ ). Define $\mathfrak{a}$ as the fractional ideal of $K$ such that $\mathfrak{a} \otimes \mathcal{O}_{S, s}=a_{s} \mathcal{O}_{S, s}$ for all $s \in S \backslash U$ and $=\mathcal{O}_{S, s}$ for $s \in U$. Define similarly $\mathfrak{b}$. These ideals depend on the choice of Equation (8) and on $D$, but don't depend on the choice of $U$. Their classes in $\operatorname{Pic}(S)$ don't depend on the choice of Equation (8) over $K$.

Lemma 3.9. Let $\mathscr{C}$ be a Weierstrass model of $C$ over $S$. Let $D \in \mathscr{Z}(S)$. Let $F=\phi_{*} \mathcal{O}_{\mathscr{Z}}(D)$ and let $N$ be as in Lemma 3.1.

(1) We have

$$
\operatorname{det} F \simeq \mathfrak{a}^{-1}, \quad N \simeq \mathfrak{b}^{-1}
$$

and

$$
\Delta_{\mathscr{C}} \simeq\left(\mathfrak{a}^{g+1} \mathfrak{b}^{-2}\right)^{2(2 g+1)}, \quad \operatorname{det} f_{*} \omega_{\mathscr{C} / S} \simeq \mathfrak{a}^{-g(g+1) / 2} \mathfrak{b}^{g} .
$$

(2) Let $[\mathfrak{w}]$ be the Weierstrass class of $\mathscr{C}$ (Definition 3.3). Then

$$
\Delta_{\mathscr{C}} \simeq[\mathfrak{w}]^{\otimes 2(2 g+1)}, \quad \operatorname{det} f_{*} \omega_{\mathscr{C} / S} \simeq[\mathfrak{w}]^{\otimes(-g / 2)}
$$

if $g$ is even, and

$$
\Delta_{\mathscr{C}} \simeq[\mathfrak{w}]^{\otimes 4(2 g+1)}, \quad \operatorname{det} f_{*} \omega_{\mathscr{C} / S} \simeq[\mathfrak{w}]^{\otimes(-g)}
$$

if $g$ is odd.

(3) In $\operatorname{Pic}(S)$, we have the following equalities of subgroups

$$
\left\langle[\mathfrak{w}]^{2}\right\rangle=\left\langle\Delta_{\mathscr{C}}, \operatorname{det} f_{*} \omega_{\mathscr{C} / S}\right\rangle
$$

if $4 \mid g$, and

$$
\langle[\mathfrak{w}]\rangle=\left\langle\Delta_{\mathscr{C}}, \operatorname{det} f_{*} \omega_{\mathscr{C} / S}\right\rangle
$$

otherwise.

Proof. Part (2) follows immediately from (1) and implies Part (3). Let us prove Part (1). Keep the notation of Lemma 3.7. We choose the equation (8) with coefficients in $\mathcal{O}_{S}(U)$ and having good reduction over $U$ for some dense open subset $U \subseteq S$. We also translate $x$ and $y$ so that $x=a_{s} x_{s}$ and $y=b_{s} y_{s}$ at $s \in S \backslash U$.

We have

$$
\phi_{*} \mathcal{O}_{\mathscr{Z}}(D)(U)=\mathcal{O}_{S}(U) \oplus \mathcal{O}_{S}(U) x, \quad \phi_{*} \mathcal{O}_{\mathscr{Z}}(D)_{s}=\mathcal{O}_{S, s} \oplus \mathcal{O}_{S, s} x_{s},
$$

so we have canonically

$$
(\operatorname{det} F)(U)=\mathcal{O}_{S}(U)(1 \wedge x), \quad(\operatorname{det} F)_{s}=\mathcal{O}_{S, s}\left(1 \wedge x_{s}\right) .
$$

Then the relations $x=a_{s} x_{s}$ imply that $\operatorname{det} F \simeq \mathfrak{a}^{-1}$. By the computations in the proof of Lemma 3.1. we see that $N$ has bases $y$ over $\mathcal{O}_{S}(U)$ and $y_{s}$ over $\mathcal{O}_{S, s}$. The relations $y=b_{s} y_{s}$ then imply that $N \simeq \mathfrak{b}^{-1}$.

Denote by $\Delta$ the discriminant of Equation (8) and by $\Delta_{s}$ that of Equation (9). Then

$$
\Delta=b_{s}^{4(2 g+1)} a_{s}^{-2(g+1)(2 g+1)} \Delta_{s}
$$


(see 6], bottom of page 4581). This proves that

$$
\Delta R=\mathfrak{b}^{4(2 g+1)} \mathfrak{a}^{-2(g+1)(2 g+1)} \Delta_{\mathscr{C}}
$$

as fractional ideals of $K$, hence

$$
\Delta_{\mathscr{C}} \simeq\left(\mathfrak{a}^{g+1} \mathfrak{b}^{-2}\right)^{2(2 g+1)} .
$$

Now we compute $f_{*} \omega_{\mathscr{C} / S}$. Denote by

$$
\omega_{U}=\frac{d x}{(2 y+Q(x))} \wedge \ldots \wedge \frac{x^{g-1} d x}{(2 y+Q(x))}
$$

and

$$
\omega_{s}=\frac{d x_{s}}{\left(2 y_{s}+Q_{s}\left(x_{s}\right)\right)} \wedge \ldots \wedge \frac{x_{s}^{g-1} d x_{s}}{\left(2 y_{s}+Q\left(x_{s}\right)\right)} .
$$

They are respectively bases of $\left(\operatorname{det} f_{*} \omega_{\mathscr{C} / S}\right)(U)$ and of $\left(\operatorname{det} f_{*} \omega_{\mathscr{C} / S}\right)_{s}$. As $x=a_{s} x_{s}$ and $y=b_{s} y_{s}$, we have $Q_{s}\left(x_{s}\right)=Q(x) / b_{s}$. Thus

$$
\frac{x^{i} d x}{2 y+Q(x)}=\left(a_{s}^{i+1} b_{s}^{-1}\right) \frac{x_{s}^{i} d x_{s}}{2 y_{s}+Q\left(x_{s}\right)}
$$

and $\omega_{U}=a_{s}^{g(g+1) / 2} b_{s}^{-g} \omega_{s}$, so $\operatorname{det} f_{*} \omega_{\mathscr{C} / S} \simeq \mathfrak{a}^{-g(g+1) / 2} \mathfrak{b}^{g}$.

Corollary 3.10. Let $\mathscr{C}$ be a Weierstrass model of $C$ over $S$. Then the following properties are true.

(1) If $g=1$, then $\Delta_{\mathscr{C}} \simeq\left(\operatorname{det} f_{*} \omega_{\mathscr{C} / S}\right)^{\otimes(-12)}$ (well-known);

(2) If $g=2$, then $\Delta_{\mathscr{C} / S} \simeq\left(\operatorname{det} f_{*} \omega_{\mathscr{C} / S}\right)^{\otimes(-10)}$.

(3) If $g=4$, then $\Delta_{\mathscr{C} / S} \simeq\left(\operatorname{det} f_{*} \omega_{\mathscr{C} / S}\right)^{\otimes(-9)}$.

(4) In $\operatorname{Pic}(S), \Delta_{\mathscr{C}}$ is a $4(2 g+1)$-th power (resp. $2(2 g+1)$-th power) if $g$ is odd (resp. even).

Theorem 3.11. Let $\mathscr{C}$ be a Weierstrass model of $C$ over $S$. Then $\mathscr{C}$ is defined by an integral Weierstrass equation (Definition 2.1) under any of the following conditions:

(1) $\Delta_{\mathscr{C}}$ is principal, det $f_{*} \omega_{\mathscr{C} / S}$ is free and either

(a) $g$ is odd and $\mathscr{Z} \simeq \mathbb{P}_{S}^{1}$ or,

(b) $g \equiv 2 \bmod 4$

(so the converse of Proposition 2.6 is true if $4 \nmid g$ );

(2) $\operatorname{Pic}(S)$ is finite of odd order, $\Delta_{\mathscr{C}}$ is principal and $\operatorname{det} f_{*} \omega_{\mathscr{C} / S}$ is free;

(3) $\operatorname{Pic}(S)$ is finite of order prime to $2(2 g+1)$, or $g$ is even and the torsion elements of $\operatorname{Pic}(S)$ are of order prime to $2(2 g+1)$, and $\Delta_{\mathscr{C}}$ is principal.

(4) $\operatorname{Pic}(S)$ is finite of order prime to $2 g$ and $\operatorname{det} f_{*} \omega_{\mathscr{C} / S}$ is free.

Proof. We have to show that $\mathscr{Z} \simeq \mathbb{P}_{S}^{1}$ and $[\mathfrak{w}]$ is trivial under any of the conditions in the theorem.

(1.a) This follows from Lemma 3.9 (3).

(1.b) Take any section $D \in \mathscr{Z}(S)$ and consider the fractional ideals $\mathfrak{a}, \mathfrak{b}$ as in Definition 3.8. Then Lemma3.9 (1) implies that $\mathfrak{a}^{-g(g+1) / 2} \mathfrak{b}^{g} \simeq \mathcal{O}_{S}$, so $\mathfrak{a}$ is a square, and $\mathscr{Z} \simeq \mathbb{P}_{S}^{1}$. Lemma $[3.9(3)$ implies that $[\mathfrak{w}]=1$.

(2) First we have $\mathscr{Z} \simeq \mathbb{P}_{S}^{1}$ by Proposition 1.4. Lemma 3.9)(3) implies that $[\mathfrak{w}]^{2}=1$, hence $[\mathfrak{w}]=1$. 
(3) If $\operatorname{Pic}(S)$ is finite of order prime to $2(2 g+1)$, then $\mathscr{Z} \simeq \mathbb{P}_{S}^{1}$. Therefore $[\mathfrak{w}]^{4(2 g+1)}=1$ by Lemma $3.9(2)$. Thus $[\mathfrak{w}]=1$.

Now suppose $g$ is even and the torsion elements of $\operatorname{Pic}(S)$ are of order prime to $2(2 g+1)$. With the notation of Lemma 3.9, as $\Delta_{\mathscr{C}}$ is principal, $\mathfrak{a}^{g+1} \mathfrak{b}^{-2}$ is a torsion element of order dividing $2(2 g+1)$, therefore trivial. So $\mathfrak{a}$ is a square in $\operatorname{Pic}(S)$ as $g$ is even, and $\mathscr{Z} \simeq \mathbb{P}_{S}^{1}$. We can now argue as in the first part.

(4) Similar to (3).

As an immediate consequence, we proved a conjecture of M. Sadek [10].

Corollary 3.12. Let $K$ be a number field of class number prime to $2(2 g+1)$. Let $C$ be a hyperelliptic curve of genus $g$ having everywhere good reduction over $K$. Then the smooth model $\mathscr{C}$ of $C$ is defined by an integral Weierstrass equation.

Proof. By the uniqueness of the smooth projective model, the hyperelliptic involution of $C$ extends to an involution on $\mathscr{C}$ and the quotient is smooth because $\mathscr{C}$ is a smooth curve. So $\mathscr{C}$ is a Weierstrass model of $C$ with $\Delta_{\mathscr{C}}=\mathcal{O}_{K}$. By Theorem $3.11(3), \mathscr{C}$ is defined by an integral Weierstrass equation.

\section{Pointed integral Weierstrass equations}

The usual Weierstrass models of elliptic curves $E$ are pointed Weierstrass models with respect to the origin $o$ of $E$, in the sense that $o$ extends to a section contained in the smooth locus of the Weierstrass model. For hyperelliptic curves $C$ having a rational Weierstrass point $P \in C(K)$ (that is $\left.\operatorname{dim}_{K} H^{0}\left(C, \mathcal{O}_{C}(2 P)\right)=2\right)$, P. Lockhart ([8]) defined and studied pointed integral Weierstrass equations for $(C, P)$.

Definition 4.1 A pointed Weierstrass model of $(C, P)$ over $S$ is a Weierstrass model $\mathscr{C}$ such that the Zariski closure $\overline{\{P\}}$ is contained in the smooth locus of $\mathscr{C} \rightarrow S$. Such a model is said to be defined by a pointed integral Weierstrass equation of $(C, P)$ if the affine scheme $\mathscr{C} \backslash \overline{\{P\}}$ is defined by

$$
y^{2}+Q(x) y=P(x)
$$

with $P(x), Q(x) \in \mathcal{O}_{S}[x], P(x)$ is monic of degree $2 g+1$ and $\operatorname{deg} Q(x) \leq g$.

Note that it may happen that a pointed Weierstrass model $\mathscr{C}$ is defined by an integral Weierstrass equation, but not by a pointed integral Weierstrass equation (see Proposition 6.1(e)).

We also have an obvious notion of minimal pointed Weierstrass model. Such a model always exists and is unique up to isomorphism (Corollary 5.2). When $\mathcal{O}_{S}$ is principal (e.g. a discrete valuation ring), the minimal pointed Weierstrass model is defined by a pointed integral Weierstrass equation (see e.g., [8], Proposition 2.8).

Let $\mathscr{C}$ be a pointed Weierstrass model of $(C, P)$. Let us recall the definition of the pointed Weierstrass class $[\mathfrak{u}] \in \operatorname{Pic}(S)$ of $\mathscr{C}$ similarly to [8], Definition 2.7. Let

$$
y^{2}+Q(x) y=P(x)
$$

be a pointed Weierstrass equation of $(C, P)$ over $K$, of discriminant $\Delta \in K^{*}$. For any closed point $s \in S$, we have a pointed integral Weierstrass equation

$$
y_{s}^{2}+Q_{s}\left(x_{s}\right) y_{s}=P_{s}\left(x_{s}\right)
$$

for $\mathscr{C} \times{ }_{S} \operatorname{Spec} \mathcal{O}_{S, s}$, with discriminant $\Delta_{s} \in \mathcal{O}_{S, s} \backslash\{0\}$. Using Lemma 3.7, we see that translating, if necessary, $x$ and $x_{s}$ by elements of $K$ and $\mathcal{O}_{S, s}$ respectively and 
$y, y_{s}$ by polynomials of degree $\leq g$ in $K[x]$ and in $\mathcal{O}_{S, s}[x]$ respectively (which keeps the equations as pointed Weierstrass equations), we can suppose that

$$
x=a_{s} x_{s}, \quad y=b_{s} y_{s}, \quad a_{s}, b_{s} \in K^{*} .
$$

As $P(x)$ and $P_{s}\left(x_{s}\right)$ are monic of degree $2 g+1$ we find $a_{s}^{2 g+1}=b_{s}^{2}$. Denote by $u_{s}=a_{s}^{g} / b_{s}$, then $a_{s}=u_{s}^{-2}$ and $b_{s}=u_{s}^{-(2 g+1)}$.

Definition 4.2 (8), Definition 2.7) The class in $\operatorname{Pic}(S)$ of the fractional ideal $\mathfrak{u}$ of $K$ such that $\mathfrak{u} \otimes \mathcal{O}_{S, s}=u_{s} \mathcal{O}_{S, s}$ for all $s \in S$ is called the pointed Weierstrass clas $₫$ of $\mathscr{C}$. It does not depend on the choice of the equation (10). With the notation of Definition 3.8 , if we take for $D$ the image of $\overline{\{P\}}$ in $\mathscr{Z}(S)$, we have $\mathfrak{a}=\mathfrak{u}^{-2}$ and $\mathfrak{b}=\mathfrak{u}^{-(2 g+1)}$.

Note that $[\mathfrak{u}]$ differs from the non-pointed Weierstrass class (Definition 3.3). Using Lemma 3.9 (1), we see that $[\mathfrak{w}]=[\mathfrak{u}]^{g}$ if $g$ is odd, and $[\mathfrak{w}]=[\mathfrak{u}]^{2 g}$ if $g$ is even.

Proposition 4.3. Let $\mathscr{C}$ be a pointed Weierstrass model of $(C, P)$. Let $D \in \mathscr{Z}(S)$ be the image of $\overline{\{P\}} \in \mathscr{C}(S)$.

(1) We have

$$
\Delta_{\mathscr{C}} \simeq \mathfrak{u}^{4 g(2 g+1)}, \quad \operatorname{det} f_{*} \omega_{\mathscr{C} / S} \simeq \mathfrak{u}^{-g^{2}} .
$$

(2) The model $\mathscr{C}$ is defined by a pointed integral Weierstrass equation if and only if $\mathfrak{u}$ is principal.

(3) The quotient $\mathscr{Z}$ is isomorphic to $\mathbb{P}_{S}^{1}$.

Proof. (1) Follows from Lemma 3.9. See also 8], Equality (2.3) and Proposition 1.12 .

(2) The only if part is clear. Suppose conversely that $\mathfrak{u}$ is principal. Then $\mathfrak{a}$ is trivial and $D$ is an elementary section. As $\mathfrak{b}$ is principal, $\mathscr{C}$ is defined by an integral Weierstrass equation by Step (1) in the proof of Proposition 3.4

(3) We use Lemma 3.9. As $\mathfrak{a}=\mathfrak{u}^{-2}$ is a square in $\operatorname{Pic}(S)$ and $\operatorname{det} \phi_{*} \mathcal{O}_{\mathscr{Z}}(D) \simeq \mathfrak{a}^{-1}$ by Lemma 3.9 (1), we find $\mathscr{Z} \simeq \mathbb{P}_{S}^{1}$ (Proposition 1.4).

Corollary 4.4. If $\mathrm{Pic}(S)$ has finite order prime to $2 g(2 g+1)$ and if $\Delta_{\mathscr{C}}$ is principal (e.g. if $\mathscr{C}$ is smooth over $S$ ), then $\mathscr{C}$ is defined by a pointed integral Weierstrass equation.

The next proposition is a straightforward generalization of [1], Theorem 5 .

Proposition 4.5. Let $(C, P)$ be a pointed hyperelliptic curve over a number field $K$. Let $\mathfrak{u}$ be any fractional ideal of $K$. Then $[\mathfrak{u}]$ is the pointed Weierstrass class of the minimal pointed Weierstrass model of some quadratic twist of $(C, P)$.

Proof. The proof is the same as for [11, Theorem 5. The only point we have to check is that the minimal pointed Weierstrass model commutes with étale base changes in higher genus. See Proposition 5.4. Note that, in general, this is false for non-pointed minimal Weierstrass models in higher genus ([], Proposition 4, p. $4595)$.

Remark 4.6 A referee raised the natural question to study, for each class [a] in $\mathrm{Cl}(K)$, the density of the hyperelliptic curves over $K$ having (pointed or not) Weierstrass class equal to $[\mathfrak{a}]$, indicating that this question is solved for elliptic curves in 2]. Recent related works can be found in $[3]$.

\footnotetext{
${ }^{1}$ In fact our definition is the inverse of that of $[8]$.
} 


\section{Complements on minimal Pointed Weierstrass models}

We prove the uniqueness of the minimal pointed Weierstrass model (see also Remark 5.3) and its compatibility with étale base changes. These properties are local on $S=\operatorname{Spec} R$.

Suppose first that $R$ is a discrete valuation ring with uniformizing element $t$, residue field $k$ and normalized valuation $v$. Let $\mathscr{C}, \mathscr{C}^{\prime}$ be two distinct Weierstrass models of $C$ over $S$, with respective quotients $\mathscr{Z}, \mathscr{Z}^{\prime}$ by the hyperelliptic involution. Up to actions of $\mathrm{Gl}_{2}(R)$, there are parameters $x, x^{\prime}$ of $\mathscr{Z}, \mathscr{Z}^{\prime}$ respectively and $d>0$ such that $x=t^{d} x^{\prime}$. Let $p \in \mathscr{C}_{k}$ be a rational point over the zero of $x$ in $\mathscr{Z}_{k}$.

Lemma 5.1. If $\mathscr{C}$ is regular at $p$, then $v\left(\Delta_{\mathscr{C}}\right)<v\left(\Delta_{\mathscr{C}^{\prime}}\right)$.

Proof. This is a special case of [6], Proposition 3 (iii)-(iv), p. 4594. However, op. cit. is written under the hypothesis that $k$ is perfect. So we give an ad hoc proof here.

We suppose $\operatorname{char}(k)=2$. The other cases are similar and easier to deal with. For $0 \leq i \leq d$, denote by $\mathscr{Z}_{i}$ the smooth model of $\mathbb{P}_{K}^{1}$ with parameter $x_{i}=x / t^{i}$ and by $\mathscr{C}_{i}$ the normalization of $\mathscr{Z}_{i}$ in $K(C)$. So $\mathscr{C}=\mathscr{C}_{0}$ and $\mathscr{C}^{\prime}=\mathscr{C}_{d}$. We are going to prove that $v\left(\Delta_{\mathscr{C}_{i}}\right) \leq v\left(\Delta_{\mathscr{C}_{i+1}}\right)$ for any $0 \leq i \leq d-1$ with strict inequality when $i=0$.

Let

$$
y^{2}+\left(a_{1}+a_{3} x+\ldots\right) y=t a_{0}+a_{2} x+a_{4} x^{2}+\ldots, \quad a_{i} \in R
$$

be an affine equation of $\mathscr{C}$ with $p$ corresponding to $x=y=0$. As $\mathscr{C}$ is regular at $p$, at least one of $a_{0}, a_{1}, a_{2}$ is a unit in $R$. Using [6], Lemme 2, p. 4582, we verify that the equation

$$
y^{2}+\left(a_{1}+t a_{3} x_{1}+\ldots\right) y=t\left(a_{0}+a_{2} x_{1}+t a_{4} x_{1}^{2}+\ldots\right) .
$$

defines an affine normal scheme, hence it is an affine equation of $\mathscr{C}_{1}$ and we have

$$
v\left(\Delta_{\mathscr{C}_{1}}\right)=v\left(\Delta_{\mathscr{C}}\right)+2(2 g+1)(g+1)>v\left(\Delta_{\mathscr{C}}\right) .
$$

Suppose that $d \geq 2$. Let $p_{1} \in \mathscr{C}_{1}(k)$ be a point corresponding to $x_{1}=0$. Then $\mathscr{C}_{1}$ is regular at $p_{1}$ if either $a_{0}$ or $a_{1} \in R^{*}$ and we can then continue with induction. If $a_{0}, a_{1} \in t R$ and $a_{2} \in R^{*}$, then the above equation becomes

$$
y_{1}^{2}+\left(t^{-1} a_{1}+t a_{3} x_{2}+\cdots\right) y_{1}=t^{-1} a_{0}+a_{2} x_{2}+t^{2} a_{4} x_{2}^{2}+\cdots,
$$

where $y_{1}=y / t$. Again this defines a normal scheme, hence $\mathscr{C}_{2}$. We have $v\left(\Delta_{\mathscr{C}_{1}}\right)=$ $v\left(\Delta_{\mathscr{C}_{2}}\right)+2(2 g+1)(g-1) \geq v\left(\Delta_{\mathscr{C}_{2}}\right)$. As $\mathscr{C}_{2}$ is smooth at $x_{2}=0$, we can also continue with induction if $d \geq 3$.

Corollary 5.2. Let $C$ be a hyperelliptic curve over $K$ and let $P \in C(K)$ be a Weierstrass point. Then $(C, P)$ admits a unique minimal pointed Weierstrass model of $(C, P)$ over $S$.

Proof. The existence is obvious when $S$ is local. In the general case $C$ has good reduction over a dense open subset $U$ of $S$. Then it is enough to glue local minimal pointed Weierstrass models over the points of $S \backslash U$ with the smooth model over $U$.

Now we prove the uniqueness. One can suppose $S$ is local. Let $\mathscr{C}, \mathscr{C}^{\prime}$ be two distinct pointed Weierstrass models of $(C, P)$. We keep the notation preceding Lemma 5.1. Let $p_{0}$ be the specialization of $P$ in $\mathscr{C}_{k}$. This is a smooth point by definition. If $p_{0}=p$, then $v\left(\Delta_{\mathscr{C}}\right)<v\left(\Delta_{\mathscr{C}^{\prime}}\right)$ by the same lemma. Otherwise, 
$x(P) \notin t R$ (may be $\infty$ ). This implies that $\left(x^{\prime}\right)^{-1}(P)=t^{d} x(P)^{-1} \in t R$. Hence the specialization of $P$ in $\mathscr{Z}_{k}^{\prime}$ is the zero of $x^{-1}=t^{d} x^{-1}$ and Lemma 5.1 implies that $v\left(\Delta_{\mathscr{C}^{\prime}}\right)<v\left(\Delta_{\mathscr{C}}\right)$. In other words, two distinct pointed Weierstrass models always have distinct discriminant ideals. Therefore the minimal one is unique.

Remark 5.3 This corollary also follows from [8, Remark after Definition 2.1 (but whose proof is left to readers). Note that the non-pointed minimal Weierstrass model is not unique in general even when $C$ has a rational Weierstrass point.

Proposition 5.4 (Étale base change). Let $(C, P)$ be a pointed hyperelliptic curve over $K$. Let $\mathscr{C}$ be the minimal pointed Weierstrass model of $(C, P)$ over $S$. Let $S^{\prime} \rightarrow S$ be an étale morphism of affine Dedekind schemes and let $K^{\prime}=K\left(S^{\prime}\right)$. Then $\mathscr{C} \times{ }_{S} S^{\prime}$ is the minimal pointed Weierstrass model of $\left(C_{K^{\prime}}, P\right)$ over $S^{\prime}$.

Proof. The property is local on $S^{\prime}$, so we can suppose that $S$ and $S^{\prime}=\operatorname{Spec} R^{\prime}$ are local. The base change $\mathscr{C} \times{ }_{S} S^{\prime}$ is a normal scheme because $S^{\prime} / S$ is étale. So $\mathscr{C} \times{ }_{S} S^{\prime}$ is a pointed Weierstrass model of $\left(C_{K^{\prime}}, P\right)$.

(1) First suppose that the residue extension of $S^{\prime} / S$ is trivial. Then $R$ is dense in $R^{\prime}$. Let $\mathscr{C}^{\prime}$ be a pointed Weierstrass model over $S^{\prime}$. We will show that it is defined over $S$. Let $\mathscr{Z}^{\prime}$ be its quotient by the hyperelliptic involution. Let $x^{\prime}$ be a parameter of $\mathscr{Z}^{\prime}$ whose pole divisor is the Zariski closure $\overline{\{Q\}} \subset \mathscr{Z}^{\prime}$, where $Q$ is the image of $P$ in $\mathbb{P}_{K}^{1}(K)$. As $R$ is dense in $R^{\prime}$, we can find $x \in K\left(\mathbb{P}_{K}^{1}\right)$ such that $x^{\prime}=x+a^{\prime} x^{\prime}+b^{\prime}$ with $a^{\prime}, b^{\prime} \in \mathfrak{m}_{R^{\prime}}$. Thus $R^{\prime}\left[x^{\prime}\right]=R^{\prime}[x]=R[x] \otimes_{R} R^{\prime}$ and $\mathscr{Z}^{\prime}=\mathscr{Z} \times{ }_{S} S^{\prime}$ for some smooth model $\mathscr{Z}$ of $\mathbb{P}_{K}^{1}$. The normalization $\mathscr{C}_{0}$ of $\mathscr{Z}$ in $K(C)$ is a pointed Weierstrass model of $(C, P)$ over $S$ and $\mathscr{C}_{0} \times_{S} S^{\prime}$ is equal to $\mathscr{C}^{\prime}$ because the former is normal. Therefore

$$
v\left(\Delta_{\mathscr{C}^{\prime}}\right)=v\left(\Delta_{\mathscr{C}_{0}}\right) \geq v\left(\Delta_{\mathscr{C}}\right)=v\left(\Delta_{\mathscr{C} \times S S^{\prime}}\right)
$$

and $\mathscr{C} \times{ }_{S} S^{\prime}$ is minimal.

(2) In the general case, we can first replace $R$ with its henselization (the first step shows that $\mathscr{C}$ commutes with henselization of $R$ ). The integral closure of $R$ in the Galois closure of $K^{\prime} / K$ is a discrete valuation ring unramified over $R$. So we can suppose that $S^{\prime} / S$ is Galois with Galois group $\Gamma$. Let $\mathscr{C}^{\prime}$ be the minimal pointed Weierstrass model of $\left(C_{K^{\prime}}, P\right)$ over $S^{\prime}$. As $P$ is invariant by $\Gamma$ and the minimal pointed Weierstrass model is unique, $\Gamma$ acts on $\mathscr{C}^{\prime}$ (as $S$-scheme) and on the quotient of $\mathscr{C}^{\prime}$ by the hyperelliptic involution. The quotient $\mathscr{C}_{0}:=\mathscr{C}^{\prime} / \Gamma$ is a Weierstrass model of $C$ over $S$. As $S^{\prime} / S$ is étale, $\mathscr{C}_{0} \times{ }_{S} S^{\prime}$ is normal and $\mathscr{C}^{\prime} \rightarrow \mathscr{C}_{0} \times{ }_{S} S^{\prime}$ is an isomorphism. Hence $\mathscr{C}$ is smooth along the Zariski closure of $P$ and is a pointed Weierstrass model of $(C, P)$. Moreover, $v\left(\Delta_{\mathscr{C}^{\prime}}\right)=v\left(\Delta_{\mathscr{C}_{0}}\right) \geq v\left(\Delta_{\mathscr{C}}\right)$. So $\mathscr{C}^{\prime} \simeq \mathscr{C} \times{ }_{S} S^{\prime}$.

\section{EXAMPLES}

We construct examples of Weierstrass models not defined by integral (or pointed integral) Weierstrass equations, but with various nice properties. We will work with number fields $K$. Let us summarize first the examples we will construct.

Proposition 6.1. There exist number fields $K$, hyperelliptic curves $C$ over $K$ with Weierstrass model $\mathscr{C}$ over $S=\operatorname{Spec} \mathcal{O}_{K}$, of any of the following types:

(a)

$$
\mathscr{Z} \nsucceq \mathbb{P}_{S}^{1}, \quad \Delta_{\mathscr{C}} \simeq \mathcal{O}_{K}, \quad \operatorname{det} f_{*} \omega_{\mathscr{C} / S} \simeq \mathcal{O}_{S} ;
$$


(b)

(c)

$$
\mathscr{Z} \simeq \mathbb{P}_{S}^{1}, \quad \Delta_{\mathscr{C}} \simeq \mathcal{O}_{K}, \quad \operatorname{det} f_{*} \omega_{\mathscr{C} / S} \not \mathcal{O}_{S} ;
$$

$$
\mathscr{Z} \simeq \mathbb{P}_{S}^{1}, \quad \Delta_{\mathscr{C}} \nsucceq \mathcal{O}_{K}, \quad \operatorname{det} f_{*} \omega_{\mathscr{C} / S} \simeq \mathcal{O}_{S} ;
$$

(by Proposition [2.6, in all the above situations, $\mathscr{C}$ is not defined by an integral Weierstrass equation.)

(d)

$$
\mathscr{Z} \simeq \mathbb{P}_{S}^{1}, \quad \Delta_{\mathscr{C}} \simeq \mathcal{O}_{K}, \quad \operatorname{det} f_{*} \omega_{\mathscr{C} / S} \simeq \mathcal{O}_{S}
$$

but $\mathscr{C}$ is not defined by an integral Weierstrass equation;

(e) $\mathscr{C}$ is a minimal pointed Weierstrass model, defined by an integral Weierstrass equation but not by a pointed integral Weierstrass equation.

Remark 6.2 The genus $g$ of the curves $C$ appearing in our construction is not arbitrary. For (a) and for (b) there are infinitely many possible $g$ (always odd). If we relax the condition $\mathscr{Z} \simeq \mathbb{P}_{S}^{1}$ in (b), we find all odd integers $g \geq 5$ except those of the form $2^{n}-1$. For (c), $g$ can be any integer different from 1,2 and 4 . For (d), $g$ can be any integer divisible by 4 . Finally, for (e), $g$ can be any integer $\geq 2$.

Example 6.3 Fix $g_{0} \geq 1$. We start with a hyperelliptic curve $C_{0}$ of genus $g_{0} \geq 1$ over a number field $K$ with everywhere good reduction. Such a curve always exists by enlarging $K$ if necessary 2 Let $\mathscr{C}_{0}$ be its smooth projective model over $S=$ Spec $\mathcal{O}_{K}$, and let $\mathscr{Z}_{0}$ be the corresponding projective bundle over $S$. Extending $K$ if necessary, we can suppose that $\mathscr{Z}_{0} \simeq \mathbb{P}_{S}^{1}$. Let $B \subset \mathscr{Z}_{0}$ be the branch locus of $\mathscr{C}_{0} \rightarrow \mathscr{Z}_{0}$. This is a closed subset finite over $S$. By [4, Theorem 7.2, there exists an integral point $D_{0}$ in $\mathscr{Z}_{0} \backslash B$. Applying again the theorem to $\mathscr{Z}_{0} \backslash\left(B \cup D_{0}\right)$, we find an integral point $D_{\infty}$ such that $B, D_{0}$ and $D_{\infty}$ are pairwise disjoint. Extending $K$ if necessary, we can suppose that $\mathscr{C}_{0}$ is defined by an integral Weierstrass equation

$$
y^{2}+Q(x) y=P(x),
$$

and $D_{0}, D_{\infty}$ are respectively the zero and pole divisors of $x$. Write

$$
4 P(x)+Q(x)^{2}=a_{0} x^{2 g_{0}+2}+\cdots+a_{2 g_{0}+2} \in \mathcal{O}_{K}[x] .
$$

As $\left(D_{0} \cup D_{\infty}\right) \cap B=\emptyset$, we have $a_{0}, a_{2 g_{0}+2} \in \mathcal{O}_{K}^{*}$.

Extending again $K$, we can suppose that the class group $\operatorname{Pic}(S)$ of $K$ contains a maximal cyclic 2-subgroup $H_{2} \subseteq \operatorname{Pic}(S)$ of order $\geq 4$ (see Lemma 6.5 below). Let $[I] \in H_{2}$ (it will be chosen more precisely later). We will use $[I]$ to construct a hyperelliptic curve $C$ over $K$ together with a Weierstrass model $\mathscr{C}$ as double coverings of $\mathscr{C}_{0}$. Let $d$ be the order of $[I]$. It is a power of 2 . As $I^{d}$ is principal, we identify $\mathbb{P}\left(\mathcal{O}_{K} \oplus I^{d}\right)$ with $\mathscr{Z}_{0}$, and the sections corresponding to the projections of $\mathcal{O}_{K} \oplus I^{d} \rightarrow \mathcal{O}_{K}$ and $\mathcal{O}_{K} \oplus I^{d} \rightarrow I^{d}$ respectively to $D_{0}$ and $D_{\infty}$. Consider now $\mathscr{Z}:=\mathbb{P}\left(\mathcal{O}_{K} \oplus I\right)$ and the canonical morphism

$$
\theta: \mathbb{P}\left(\mathcal{O}_{K} \oplus I\right) \rightarrow \mathbb{P}\left(\mathcal{O}_{K} \oplus I^{d}\right)=\mathscr{Z}_{0} .
$$

\footnotetext{
${ }^{2}$ For some special values of $g_{0}$, we can give explicit examples as follows. Let $r \geq 3$ be an odd square-free integer. Consider the hyperelliptic curve $C_{0}$ over $\mathbb{Q}$ defined by $y^{2}+y=x^{r}$. If $\xi_{r} \in \mathbb{C}$ is a primitive $r$-th root of the unity and if $m \in \mathbb{Z}$ satisfies $4 m \equiv-1 \bmod r^{(r+1) / 2}$, then $C_{0}$ has everywhere good reduction over $K=\mathbb{Q}\left[m^{1 / r}, \sqrt{1-\xi_{r}}\right]$. The corresponding smooth model $\mathscr{C}_{0}$ is defined by an integral global Weierstrass equation, and $\left(1-\xi_{r}\right)^{-1}\left(x-m^{1 / r}\right)$ is a parameter of $\mathscr{Z}_{0}$.
} 
Locally on $S$ it corresponds to taking the $d$-th powers of the homogeneous coordinates. Let

$$
\mathscr{C}:=\mathscr{C}_{0} \times \mathscr{Z}_{0} \mathscr{Z}
$$

Let us study some properties of $\mathscr{C}$. Let $e$ be a basis of $I^{d}$. For any open subset $U \subseteq S$ such that $\left.I\right|_{U}$ has a basis $e_{U}$, we have $e_{U}^{d}=\alpha_{U}^{-1} e$ with $\alpha_{U} \in \mathcal{O}_{S}(U)^{*}$ a unit. Then $\mathscr{C}_{U}$ is defined by the equation

$$
y^{2}+Q\left(\alpha_{U} t^{d}\right) y=P\left(\alpha_{U} t^{d}\right) .
$$

By Lemma 6.6 below, this equation defines a hyperelliptic curve $C$ over $K$ of odd genus $g=d\left(g_{0}+1\right)-1$ and the discriminant ideal $\Delta_{\mathscr{C}_{U}}$ defined by this equation satisfies

$$
\Delta_{\mathscr{C}_{U}}= \pm\left(a_{0} a_{2 g_{0}+2}\right)^{d-1} \alpha_{U}^{\left(2 g_{0}+2\right)\left(\left(2 g_{0}+2\right) d-1\right)} d^{2 g+2} \Delta_{\left(\mathscr{C}_{0}\right)_{U}}^{d}=d^{2 g+2} \mathcal{O}_{U} .
$$

Therefore $\Delta_{\mathscr{C}}=d^{2 g+2} \mathcal{O}_{K}$ and $\mathscr{C} \rightarrow S$ is smooth aways from primes dividing 2 . At any prime $\mathfrak{p}$ of $\mathcal{O}_{K}$ dividing 2 , as $Q(x)$ is non-zero in $k(\mathfrak{p})[x]$, the fiber $\mathscr{C}_{\mathfrak{p}}$ is reduced, so $\mathscr{C}$ is normal (7], Lemma 4.1.18) and is a Weierstrass model of $C$ over $S$.

Let $D$ be the section of $\mathscr{Z}$ corresponding to the projection $\mathcal{O}_{K} \oplus I \rightarrow I$. Then $\operatorname{det} \phi_{*} \mathcal{O}_{\mathscr{Z}}(D)=I$ and $\theta(D)=D_{\infty}$. As $\mathscr{C}$ is obtained by base change from $\mathscr{C}_{0}$, we have $N \simeq \mathcal{O}_{K}$ (tensor product the exact sequence (5) corresponding to $\mathscr{C}_{0}$ by $\mathcal{O}_{\mathscr{Z}}$ ). By Lemma 3.9(1), we have

$$
\operatorname{det} f_{*} \omega_{\mathscr{C} / S} \simeq I^{g(g+1) / 2} \simeq I^{d \times \frac{\left(d\left(g_{0}+1\right)-1\right)\left(g_{0}+1\right)}{2}},
$$

$[\mathfrak{w}]=[I]^{-d\left(g_{0}+1\right) / 2}$ (and also $\Delta_{\mathscr{C}} \simeq \mathcal{O}_{K}$, but this is less precise then the above equality $\left.\Delta_{\mathscr{C}}=d^{2 g+2} \mathcal{O}_{K}\right)$. Therefore:

(a) if we take for $[I]$ a generator of $H_{2}$ and if $g_{0}$ is odd, then $[I]$ is not a square, so

$$
\mathscr{Z} \not \mathbb{P}_{S}^{1}, \quad \Delta_{\mathscr{C}} \simeq \mathcal{O}_{K}, \quad \operatorname{det} f_{*} \omega_{\mathscr{C} / S} \simeq \mathcal{O}_{S}, \quad[\mathfrak{w}]=1 ;
$$

(b) if $[I]$ is the square of some element of $\mathrm{H}_{2}$ and if $g_{0}$ is even, then

$$
\mathscr{Z} \simeq \mathbb{P}_{S}^{1}, \quad \Delta_{\mathscr{C}} \simeq \mathcal{O}_{K}, \quad \operatorname{det} f_{*} \omega_{\mathscr{C} / S} \not \mathcal{O}_{S}, \quad[\mathfrak{w}] \neq 1
$$

Example 6.4 Let $g \geq 1$. By [1], there exists a quadratic field $K$ such that $\mathrm{Cl}(K)$ contains a cyclic subgroup of order $4 g^{2}$ (see the beginning of the proof of Lemma 6.5 below). Let $S=\operatorname{Spec} \mathcal{O}_{K}$ and let $[\mathfrak{u}] \in \operatorname{Pic}(S)$. By Proposition 4.5 , there exists a pointed hyperelliptic curve $(C, P)$ over $K$ such that the pointed Weierstrass class of the minimal pointed Weierstrass model $\mathscr{C}$ is equal to $[\mathfrak{u}]$.

(c) If $g \not \equiv 0 \bmod 4$, we choose [u] of order $g^{2}$. Then $\mathscr{Z} \simeq \mathbb{P}_{S}^{1}, \Delta_{\mathscr{C}}$ is not principal, and $\operatorname{det} f_{*} \omega_{\mathscr{C} / S}$ is free (Proposition 4.3(1)).

(d) If $g \equiv 0 \bmod 4$ and if we choose [u] of order $4 g$, then $\mathscr{Z} \simeq \mathbb{P}_{S}^{1}, \Delta_{\mathscr{C}}$ is principal and $\operatorname{det} f_{*} \omega_{\mathscr{C} / S}$ is free. Let $[\mathfrak{w}]$ be the Weierstrass class of $\mathscr{C}$. Then $[\mathfrak{w}]=[\mathfrak{u}]^{2 g}$ (Definition 4.2) is non-trivial. So $\mathscr{C}$ is not defined by an integral Weierstrass equation (Proposition 3.4).

(e) If $g \geq 2$, choose $[\mathfrak{u}]$ of order $g$. Then [w] is trivial. As $\mathscr{Z} \simeq \mathbb{P}_{S}^{1}$, the model $\mathscr{C}$ is defined by an integral Weierstrass equation by Proposition 3.4. But it is not defined by a pointed integral Weierstrass equation.

Note that in all the above examples except (c), $\Delta_{\mathscr{C}}$ is principal but not equal to $\mathcal{O}_{K}$ in general. 
Lemma 6.5. Let $K$ be a number field. Let $d \geq 2$. Then there exists an extension $L / K$ (of degree at most 2 ) such that the class group of $L$ contains a cyclic subgroup of order $d$.

Proof. For any $n \geq 1$, in the proof of 1 , Theorem 1, explicit quadratic extensions $F / \mathbb{Q}$ whose class groups contain a cyclic subgroup $C_{n}$ of order $n$ are constructed. Fix such an $F$ with $n=d[K: \mathbb{Q}]$. Let $L=K F$. Using the norm map $\mathrm{Cl}(L) \rightarrow$ $\mathrm{Cl}(F)$, we see that the kernel of the canonical map $\mathrm{Cl}(F) \rightarrow \mathrm{Cl}(L)$ is annihilated by $[K: \mathbb{Q}]$, so the image of $C_{n}$ in $\mathrm{Cl}(L)$ has order divisible by $d$ and we are done.

Lemma 6.6. Let

$$
E_{0}: \quad y^{2}+Q(x) y=P(x)
$$

be a Weierstrass equation of a hyperelliptic curve $C_{0}$ of genus $g_{0}$ over some field $K$ with $4 P(x)+Q(x)^{2}=a_{0} x^{2 g_{0}+2}+\cdots+a_{2 g_{0}+2}$ and $a_{0} a_{2 g_{0}+2} \neq 0$ (the latter condition means that the covering $C_{0} \rightarrow \mathbb{P}_{K}^{1}$ is unramified above the zero and pole of $x$ ). Let $\alpha \in K^{*}$ and let $d \geq 1$ be an integer prime to the characteristic of $K$. Then

$$
E_{d}: \quad y^{2}+Q\left(\alpha x^{d}\right) y=P\left(\alpha x^{d}\right)
$$

is a Weierstrass equation of a hyperelliptic curve of genus $g=d\left(g_{0}+1\right)-1$, and the discriminants of the above equations satisfy the relation

$$
\Delta\left(E_{d}\right)= \pm\left(a_{0} a_{2 g_{0}+2}\right)^{d-1} \alpha^{\left(2 g_{0}+2\right)\left(\left(2 g_{0}+2\right) d-1\right)} d^{2 g+2} \Delta\left(E_{0}\right)^{d} .
$$

Proof. (See [6], $\S 2$, for the definition of $\Delta(E)$.) This amounts to proving the following fact: if $F(x)=a_{0} x^{n}+\cdots+a_{n} \in K[x]$ has degree $n$ and $\alpha \in K^{*}$, then

$$
\operatorname{disc}\left(F\left(\alpha x^{d}\right)\right)= \pm\left(a_{0} a_{n}\right)^{d-1} \alpha^{n(n d-1)} d^{n d} \operatorname{disc}(F(x))^{d} .
$$

First by substituting $\alpha^{1 / d} x$ to $x$, we are reduced to the case $\alpha=1$. A direct computation of $\operatorname{disc}\left(F\left(x^{d}\right)\right)$ in terms of the product of the differences of the roots of $F\left(x^{d}\right)$ in some algebraic closure of $K$ leads to the desired equality.

\section{REFERENCES}

[1] N. C. Ankeny and S. Chowla: On the divisibility of the class number of quadratic fields. Pacific J. Math. 5 (1955), 321-324.

[2] E. Bekyel: The density of elliptic curves having a global minimal Weierstrass equation, J. Number Theory 109 (2004), 41-58.

[3] J. E. Cremona, M. Sadek: Local and global densities for Weierstrass models of elliptic curves, arXiv:2003.08454 [math.NT].

[4] O. Gabber, Q. Liu and D. Lorenzini: Hypersurfaces in projective schemes and a moving lemma, Duke Math. Journal, 164 (2015), 1187-1270.

[5] A. Grothendieck and J. Dieudonné: Éléments de géométrie algébrique, II. Publ. Math. IHÉS, 8 (1961).

[6] Q. Liu: Modèles entiers de courbes hyperelliptiques sur un corps de valuation discrète. Trans. of AMS, 348 (1996), 4577-4610.

[7] Q. Liu: Algebraic Geometry and Arithmetic Curves. Oxford Graduate Texts in Math., 6 (2002), 576 pages, Oxford University Press. New (corrected) edition in 2006.

[8] P. Lockhart: On the discriminant of a hyperelliptic curve. Trans. Amer. Math. Soc. 342 (1994), 729-752.

[9] K. Lønsted and S. Kleiman: Basics on families of hyperelliptic curves. Compositio Mathematica, 38 (1979), 83-111

[10] M. Sadek: Private communication (2020).

[11] J. Silverman: Weierstrass equations and the minimal discriminant of an elliptic curve. Mathematika, 31 (1984), 245-251.

[12] R. J. Stroeker: Reduction of elliptic curves over imaginary quadratic number fields. Pacific J. Math., 108 (1983), 451-463. 
[13] Stacks Project Authors: Stacks Project, stacks.math.columbia.edu, (2021).

Université de Bordeaux, Institut de Mathématiques de Bordeaux, CNRS UMr 5251, 33405 Talence, France

Email address: Qing.Liu@math.u-bordeaux.fr 